\title{
COMUNICAÇÃO
}

\section{TEN YEARS OF OCCURRENCE OF HUMAN LEISHMANIA VIANNIA BRAZILIENSIS (Lvb) INFECTIONS IN FARMS AROUND TRÊS BRAÇOS, BAHIA-BRAZIL}

\author{
J.O. Silva, E. Lago, E.F. Noronha, H.M. Okida, M.C.P. Scandiuzzi, S.I. \\ Haldi, W.D. Johnson and P.D. Marsden
}

Some years ago we published observations on the epidemiology of cutaneous leishmaniasis due to Leishmania viannia brasiliensis (Lvb) from our fiel area in Três Braços, Bahia, Brazil regarding the occurrence of human infections using techniques already described 4 . We now data of a further five years observations. The total data for 15 farms under study is set out in the Table 1 . We also crudely quantify three factors that may influence this epidemiology namely the degree of forestation of the region, the quantify of cacao grown (the chief economy of the region) and the use of insecticide (Benzene Hexachloride) to protect this crop against insect pests. Scrutinising the total number of cases for the decade there appears to be an association between prevalence of human Lvb infection and forestation as illustrated by farms 1,14 and 15. Seven farms have not indexed a case for three years. There appears to be no association with cacao growing or insecticide although the probable vector Lutzomyia whitmani is abundant in such plantations and the peridomicile ${ }^{3}$. It is possible that the surviving forest contains the original hitherto unidentified wild animal reservoir of Lvb as has been previously suggested ${ }^{2}$. Although more than 1,000 wild animals have been trapped in the area we have only recorded on Lvb in one rodent $t^{5}$. We intend to augment our field research in an attempt to identify this unknown reservoir. Although we have identified two domestic animal reservoirs in the area ${ }^{17}$, only a wild animal reservoir could be related to such an association of forest with human disease.

\section{REFERENCES}

1. Barretto AC, Cuba CC, Vexenat JA, Rosa AC, Marsden PD, Magalhães AV. Características epidemiológicas da leishmaniose tegumentar americana em uma região endêmica do Estado da Bahia. II. Leishmaniose canina. Revista da Sociedade Brasileira de Medicina Tropical 17:5965, 1984.

2. França F, Lago EL, Tada MS, Costa JML, Vale K, Oliveira J, Costa MA, Osaki M, Cheever L, Netto EM, Barreto AC, Johnson ED, Marsden PD. An outbreak of human Leishmania (viannia) braziliensis infection. Memórias do Instituto Oswaldo Cruz 86:169-174, 1991.

3. Hoch A, Ryan L, Vexenat JA, Rosa ACOC, Barretto AC. Isolation of Leishmania braziliensis

Núcleo de Medicina Tropical e Nutrição, Campus Universitário, Universidade de Brasilia.

Supported by Grant AI 16282 National Institute of Allergy and Infectious Diseases administered by the Department of International Medicine, Cornell Medical College, New York, USA.

Address to: Prof. Philip Marsden, NMTN/UnB, CP: 04671, 70919-970 Brasília, DF. braziliensis and other trypanomatids from phlebotomine in a mucocutaneous leishmaniasis endemic area, Bahia, Brazil. Memórias do Instituto Oswaldo Cruz 81(suppl): BI 44, 1986.

4. Jones TC, Johnson WD, Barretto AC, Lago E, Badaró R, Cerf B, Reed SG, Netto EM, Tada MS, França F, Wiese K, Golightly L, Fikrig E, Costa JML, Cuba CC, Marsden PD. Epidemiology of American cutaneous leishmaniasis due to Leishmania braziliensis braziliensis. Journal of Infectious Diseases 156:73-83, 1987.

5. Peterson NE, Vexemat JA, Rosa ACOC, Lago PRL. Isolation of Leishmania (viannia) braziliensis from the rodent Nectomys squamipes captured in Bahia, Brazil. Memórias do Instituto Oswaldo Cruz 83(suppl 1):BI 28,1988

6. Vexemat JA, Barretto AC, Cuba CC, Marsden PD. Características epidemiológicas da leishmaniose tegumentar americana em uma região endêmica do Estado da Bahia. III. Fauna flebotomínica. Memórias do Instituto Oswaldo Cruz 81:293-301, 1986.

7. Vexemat JA, Barretto AC, Rosa ACO, Sales CC, 
Comunicação. Silva JO, Lago E, Noronha EF, Okida HM, Scandiuzzi MCP, Haldi Sl, Johnson WD, Marsden PD. Ten years of occurrence of human Leishmania viannia braziliensis $(L v b)$ infections in farms around Três Braços, BahiaBrazil. Revista da Sociedade Brasileira de Medicina Tropical 25:81-82, jan-mar, 1992.

Magalhães AV. Infecção natural de Equus asinus por Leishmania braziliensis braziliensis - Bahia,
Brasil. Memórias do Instituto Oswaldo Cruz 81:237238, 1986.

Table 1 - Yearly occurrence of leishmaniasis in the fifteen farms.

\begin{tabular}{|c|c|c|c|c|c|c|c|c|c|c|c|c|c|c|c|c|}
\hline \multirow{2}{*}{$\begin{array}{l}\mathrm{N}^{0} \text { of } \\
\text { farm }\end{array}$} & \multirow{2}{*}{$\begin{array}{l}\text { Popula- } \\
\text { tion in } \\
1980\end{array}$} & \multicolumn{10}{|c|}{ Number of cases of leishmaniasis each year } & \multirow{2}{*}{$\begin{array}{c}\text { Total } \\
\text { cases in } \\
\text { decade }\end{array}$} & \multirow{2}{*}{$\begin{array}{l}\text { Popula- } \\
\text { tion in } \\
1989\end{array}$} & \multirow{2}{*}{$\begin{array}{c}\text { Quantity } \\
\text { of } \\
\text { forest }\end{array}$} & \multirow{2}{*}{$\begin{array}{c}\text { Quantity } \\
\text { of } \\
\text { cacao }\end{array}$} & \multirow{2}{*}{$\begin{array}{c}\text { BHC } \\
\text { use }\end{array}$} \\
\hline & & 80 & 81 & 82 & 83 & 84 & 85 & 86 & 87 & 88 & 89 & & & & & \\
\hline 1 & 224 & 3 & 1 & 1 & 0 & 2 & 0 & 1 & 6 & 1 & 0 & 15 & 215 & +++ & +++ & +++ \\
\hline 2 & 122 & 1 & 1 & 0 & 2 & 0 & 2 & 0 & 0 & 0 & 0 & 6 & 116 & ++ & +++ & +++ \\
\hline 3 & 262 & 3 & 4 & 0 & 0 & 1 & 1 & 1 & 1 & 0 & 0 & 11 & 191 & - & + & - \\
\hline 4 & 100 & 0 & 0 & 0 & 0 & 0 & 2 & 2 & 1 & 0 & 1 & 6 & 87 & + & +++ & ++ \\
\hline 5 & 107 & 3 & 2 & 1 & 0 & 1 & 0 & 1 & 1 & 1 & 0 & 10 & 129 & ++ & ++ & ++ \\
\hline 6 & 51 & 0 & 1 & 0 & 1 & 0 & 0 & 1 & 0 & 0 & 0 & 3 & 60 & + & ++ & ++ \\
\hline 7 & 100 & 2 & 1 & 0 & 0 & 0 & 0 & 0 & 0 & 0 & 0 & 3 & 115 & + & + & + \\
\hline 8 & 49 & 0 & 0 & 0 & 0 & 0 & 0 & 0 & 0 & 0 & 0 & 0 & 66 & - & + & - \\
\hline 9 & 43 & 0 & 1 & 1 & 0 & 0 & 0 & 0 & 0 & 0 & 0 & 2 & 48 & + & ++ & - \\
\hline 10 & 52 & 0 & 0 & 0 & 0 & 0 & 0 & 0 & 0 & 0 & 0 & 0 & 50 & ++ & +++ & + \\
\hline 11 & 81 & 0 & 0 & 1 & 1 & 0 & 3 & 0 & 0 & 1 & 0 & 6 & 74 & + & +++ & + \\
\hline 12 & 16 & 1 & 0 & 0 & 0 & 0 & 0 & 0 & 0 & 0 & 0 & 1 & 10 & - & ++ & - \\
\hline 13 & 111 & 0 & 1 & 0 & 0 & 0 & 0 & 0 & 0 & 0 & 2 & 3 & 84 & + & +++ . & ++ \\
\hline 14 & 131 & 0 & 3 & 0 & 1 & 0 & 1 & 2 & 3 & 3 & 4 & 17 & 146 & +++ & +++ & \\
\hline 15 & 147 & 9 & 10 & 3 & 1 & 0 & 0 & 1 & 0 & 2 & 4 & 30 & 142 & +++ & +++ & +++ \\
\hline
\end{tabular}

+ Present

++ Moderate

+++ Marked 